\title{
Extracellular vesicle-associated miR-135b and -135 a regulate stemness in Group 4 medulloblastoma cells by targeting angiomotin-like 2
}

Seung Ah Choi ${ }^{1,2}$, Eun Jung Koh ${ }^{1,3}$, Ryong Nam Kim4 ${ }^{4}$ Jung Woo Byun ${ }^{5}$, Ji Hoon Phi ${ }^{1,2}$, Jeyul Yang ${ }^{1,2}$, Kyu-Chang Wang ${ }^{1,2}$, Ae Kyung Park ${ }^{6}$, Do Won Hwang ${ }^{5}$, Ji Yeoun Lee ${ }^{1,2,7}$ and Seung-Ki Kim ${ }^{1,2^{*}}$ (D)

\begin{abstract}
Background: Extracellular vesicles (EVs) secreted by tumours, including exosomes, are important factors that regulate cell-cell interactions in oncogenesis. Although EV studies are ongoing, the biological understanding of EV-miRNAs derived from brain tumour spheroid-forming cells (BTSCs) of medulloblastoma is poor.
\end{abstract}

Purposes: We explored the specific cellular miRNAs and EV-miRNAs in medulloblastoma BTSCs to determine their potential biological function.

Methods: Bulk tumor cells (BTCs) and BTSCs were cultured under different conditions from medulloblastoma tissues $(N=10)$.

Results: Twenty-four miRNAs were simultaneously increased in both cells and EVs derived from BTSCs in comparison to BTCs. After inhibition of miR-135b or miR135a which were the most significantly increased in BTSCs, cell viability, self-renewal and stem cell marker expression decreased remarkably. Through integrated analysis of mRNAs and miRNAs data, we found that angiomotin-like 2 (AMOTL2), which was significantly decreased, was targeted by both miR-135b and miR-135a. STAT6 and GPX8 were targeted only by miR-135a. Importantly, low expression of AMOTL2 was significantly associated with overall poor survival in paediatric Group 3 and Group 4 medulloblastoma patients.

Conclusion: Our results indicated that inhibition of miR-135b or miR-135a leads to suppress stemness of BTSC through modulation of AMOTL2.

Keywords: Medulloblastoma, Brain tumour spheroid-forming cells, miR-135b, miR-135a, AMOTL2

\section{Background}

Brain tumors contain small subpopulation cells with stem cell properties that play an important role in the tumor development, progression, metastasis and recurrence $[1$,

\footnotetext{
*Correspondence: nsthomas@snu.ac.kr

${ }^{1}$ Division of Pediatric Neurosurgery, Pediatric Clinical Neuroscience Center, Seoul National University Children's Hospital, 101 Daehak-ro, Jongno-gu, Seoul 03080, Republic of Korea

Full list of author information is available at the end of the article
}

2]. These subpopulation cells have the ability spheroidforming ability and are enrichment of cancer stem cells (CSCs), also called tumor initiating cells (TICs). They are known to be capable of self-renewal and differentiation into heterogeneous cancer cell lineages [3, 4]. Studies on these cells have been reported in medulloblastoma, the most prevalent malignant pediatric brain tumor $[5,6]$.

Advances in surgery and adjuvant therapies have led to a high cure rate. Recent 5 -year survival of the patients with medulloblastoma is reaching $75 \%$. However, the

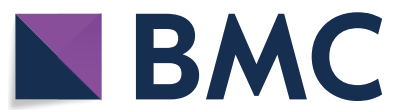

(c) The Author(s) 2020. This article is licensed under a Creative Commons Attribution 4.0 International License, which permits use, sharing, adaptation, distribution and reproduction in any medium or format, as long as you give appropriate credit to the original author(s) and the source, provide a link to the Creative Commons licence, and indicate if changes were made. The images or other third party material in this article are included in the article's Creative Commons licence, unless indicated otherwise in a credit line to the material. If material is not included in the article's Creative Commons licence and your intended use is not permitted by statutory regulation or exceeds the permitted use, you will need to obtain permission directly from the copyright holder. To view a copy of this licence, visit http://creativeco mmons.org/licenses/by/4.0/. The Creative Commons Public Domain Dedication waiver (http://creativecommons.org/publicdomain/ zero/1.0/) applies to the data made available in this article, unless otherwise stated in a credit line to the data. 
remaining $25 \%$ who do not respond to conventional therapies eventually deteriorate with recurrence and metastasis. Besides, the survivors are not free from severe adverse effects of chemotherapy and long-term sequelae of radiotherapy $[7,8]$. Therefore, we need to better understand the molecular and cellular biology of the medulloblastoma for identifying the cause of treatment resistance and developing the safe and effective treatment modality.

The clinical complexity of many brain tumors is related to the peculiar microenvironment of the central nervous system (CNS) and to its intra/inter-cellular interactions; this contributes to make brain tumor a difficult medical challenge $[9,10]$. Extracellular vesicles (EVs) are the one of forms of cell interaction that can provide an environment for cancer progression, metastasis and recurrence. Tumor-secreted EVs including exosomes play a pivotal role in the communication between tumor cells and stromal cells in local and distant microenvironments $[11,12]$. The EVs contain selected miRNAs that could regulate biological functions such as cell proliferation, differentiation, apoptosis, metabolism and drug resistance [13, 14]. Previous studies have suggested that CSC-derived EVs act as a vehicle to deliver genetic information and produce a favorable microenvironment for cancer development and they were found to contain tumor cell specific miRNAs [15].

Extensive research of molecular genetics on medulloblastoma has been done and continue to progress, but studies on EVs of the medulloblastoma remains an area of further exploration. The major EV-miRNAs and their biological roles in medulloblastoma brain tumor spheroid-forming cells (BTSCs) have not yet been determined, and only exosome studies using a few established MB cell lines and a limited number of patient serum have been reported $[10,16]$. This study was undertaken to gain insight into the identity of medulloblastoma-associated EV-miRNAs and to predict its applicability as a prognostic biomarker and therapeutic agent.

\section{Methods}

\section{Patients and samples}

Fresh tumour tissues $(\mathrm{N}=10$, Table 1$)$ were obtained from medulloblastoma patients who underwent surgery at the Seoul National University Children's Hospital. Eligible patients and/or their parents provided written informed consent to donate tumour tissue samples. The present study was approved by the Institutional Review Board (IRB) of the Seoul National University Hospital (IRB No. 1708-075-878). For primary cell culture, tumour tissues were processed within $6 \mathrm{~h}$ of resection. Snapfrozen tissues were used for subgroup analysis. Of the 10 patient samples, patient numbers 1-9 were used for all analyses, and number 10 was used only for functional analysis after validation of miR-135b, miR-135a and angiomotin-like 2 (AMOTL2) expression levels according to the analysis results.

\section{Cell cultures}

Fresh tumour tissues $(\mathrm{N}=10)$ were obtained and enzymatically dissociated to generate single cells. Single cells obtained from one patient were cultured separately into bulk tumour cells (BTCs) and brain tumour

Table 1 Patient information

\begin{tabular}{|c|c|c|c|c|c|c|c|c|}
\hline Patient no & Gender & Age & Genetic profile & Histology & M stage $^{a}$ & Location & EOR & Follow-up \\
\hline 1 & $\mathrm{~F}$ & $15 y$ & $\begin{array}{l}\text { Medulloblastoma, WNT- } \\
\text { activated }\end{array}$ & Classic & MO & Rt. Cbll & GTR & NED, alive (26 m) \\
\hline 2 & M & $8 \mathrm{y}$ & $\begin{array}{l}\text { Medulloblastoma, } \mathrm{SHH}- \\
\text { activated }\end{array}$ & Large cell/anaplastic & MO & Rt. Cbll & GTR & $\begin{array}{l}\text { Recurred at } 12 \mathrm{~m}, \mathrm{PD} \text {, expired } \\
(17 \mathrm{~m})\end{array}$ \\
\hline 3 & $\mathrm{~F}$ & $5 y$ & $\begin{array}{l}\text { Medulloblastoma, } \mathrm{SHH}- \\
\text { activated }\end{array}$ & Large cell/anaplastic & MO & Lt. Cbll & GTR & $\begin{array}{l}\text { Recurred at } 9 \text { m, PD, expired } \\
\quad(15 \mathrm{~m})\end{array}$ \\
\hline 4 & M & $5 y$ & Medulloblastoma, group 3 & With extensive nodularity & MO & $4 \mathrm{~V}$ & STR & NED, alive (48 m) \\
\hline 5 & M & $3 y$ & Medulloblastoma, group 3 & Classic & M3 & $4 \mathrm{~V}$ & GTR & $\begin{array}{l}\text { Recurred at } 9 \mathrm{~m}, \mathrm{PD} \text {, expired } \\
\quad(12 \mathrm{~m})\end{array}$ \\
\hline 6 & M & $10 y$ & Medulloblastoma, group 3 & Large cell/anaplastic & M3 & $4 \mathrm{~V}$ & NTR & Recurred at $1 \mathrm{~m}, \mathrm{PD}$, alive (42 m) \\
\hline 7 & $\mathrm{~F}$ & $12 \mathrm{y}$ & Medulloblastoma, group 4 & Large cell/anaplastic & MO & $4 \mathrm{~V}$ & NTR & NED, alive (23 m) \\
\hline 8 & $\mathrm{~F}$ & $7 y$ & Medulloblastoma, group 4 & Classic & MO & $4 \mathrm{~V}$ & NTR & NED, alive (26 m) \\
\hline 9 & M & $7 y$ & Medulloblastoma, group 4 & With extensive nodularity & M1 & $4 \mathrm{~V}$ & NTR & $\begin{array}{l}\text { Recurred at } 22 \mathrm{~m}, \mathrm{PD} \text {, expired } \\
(37 \mathrm{~m})\end{array}$ \\
\hline 10 & $\mathrm{~F}$ & $6 y$ & Medulloblastoma, group 4 & Classic & MO & $4 \mathrm{~V}$ & GTR & NED, alive (15 m) \\
\hline
\end{tabular}

Ffemale, $M$ male, yyears, MBEN medulloblastoma with extensive nodularity, Cbll cerebellum, $4 \mathrm{~V}$ fourth ventricle, EOR extent of resection, GTR gross total resection, STR subtotal resection, NTR near total resection, $m$ months, NED no evidence of disease, PD progressive disease

a M stage (Chang's classification) [47]. M0: no metastasis; M1: presence of tumor cells in the CSF; M2: nodular seeding in the cerebellar or cerebral subarachnoid space or in the third or lateral ventricle; M3: metastasis in spinal subarachnoid space; M4: metastases outside the cerebrospinal axis 
spheroid-forming cells (BTSCs) as previously described $[17,18]$. BTCs were cultured in Dulbecco's modified Eagle's medium (Invitrogen) supplemented with 10\% Exo-FBS ${ }^{\mathrm{TM}}$ Exosome-depleted foetal bovine serum (FBS; System Biosciences, Mountain View, CA). BTSCs were cultured as described previously $[17,18]$. All cells were only used in early passages $(<4)$ for experiments and were maintained at $37{ }^{\circ} \mathrm{C}$ with $5 \% \mathrm{CO}_{2}$ in a humidified atmosphere. In the additional functional study, only the BTSCs corresponding to Group 4 were used.

\section{Immunofluorescence}

The cells were plated on 8-well Lab-Tek chamber slides (Nunc), and immunofluorescence studies were carried out using the following primary antibodies: Nestin (Chemicon; 1:200) and anti-Musashi (Neuromics, Bloomington, MN; 1:100) as described previously [17]. The secondary antibodies Alexa Fluor 488-conjugated goat anti-mouse IgG and Alexa Fluor 594-conjugated goat anti-rabbit (Invitrogen; 1:200) were applied, and then the cells were counterstained with an antifading solution containing 4-6-diamidino-2-phenylindole (DAPI; Vector Laboratories). The fluorescent images were obtained with a confocal microscope (Leica, Mannheim, Germany).

\section{Isolation of EVs, including exosomes}

The EVs, including exosomes, were isolated from conditioned media using miRCURY exosome kits according to the manufacturer's instructions (Exiqon, Vedbaek, Denmark). To remove cells and cellular fragments, samples were centrifuged at $3200 \mathrm{~g}$ for $5 \mathrm{~min}$. Precipitation buffer was added to the supernatants, and EVs were precipitated by cooling at $-20^{\circ} \mathrm{C}$ for $12 \mathrm{~h}$. EV pellets collected by centrifugation at $3200 \mathrm{~g}$ for $30 \mathrm{~min}$ were dissolved in $20 \mu \mathrm{l}$ of phosphate-buffered saline (PBS).

\section{Nanoparticle tracking analysis (NTA)}

To analyse the size, concentration and distribution of EVs, NTA with a NanoSight NS3000 system (NanoSight Technology Malvern, UK) was employed. The samples were diluted to match $20-100$ objects per frame and were gently injected into the NanoSight sample chamber. The detection threshold was maintained at 7 to ensure accurate and consistent detection of small particles, as previously described [19]. The data were analysed using the NTA analytical software (ver. 2.3).

\section{Transmission electron microscopy (TEM)}

For morphology investigation, TEM (HT7700; Hitachi Ltd., Tokyo, Japan) was performed by the SNU imaging core facility. Briefly, the EVs were mixed with $4 \%$ paraformaldehyde and embedded for $20 \mathrm{~min}$ in a formvar-carbon-coated grid at room temperature. The embedded EVs were washed in PBS, fixed in $1 \%$ glutaraldehyde for $5 \mathrm{~min}$, and stained with saturated aqueous uranyl oxalate. Samples were subsequently embedded in $0.4 \%$ uranyl acetate and $1.8 \%$ methylcellulose on ice for $10 \mathrm{~min}$. Samples were dried at RT prior to visualization with TEM (JEOL, Peabody, MA). EVs were quantified by using the micro BCA protein assay (Thermo Fisher Scientific, Tokyo, Japan).

\section{Western blotting}

Proteins from cells or EVs were extracted with RIPA buffer and equal amounts of proteins were used for western blot analysis. Primary antibodies were used against Flotillin-1 (1:500, Abcam, Cambridge, MA), Calnexin (1:500, Cell Signaling Technology, Danvers, MA) and $\beta$-actin $(1: 10,000$, Sigma-Aldrich, St. Louis, MO). The membranes were incubated with a secondary antibody conjugated with horseradish peroxidase, and visualized using the enhanced Novex ${ }^{\text {TM }}$ ECL Chemiluminescent Substrate Reagent Kit (Invitrogen).

\section{NanoView analysis}

EVs were detected using the ExoView chip (NanoView Biosciences, Brighton, MA) according to the manufacturer's protocol and as previously described [20,21]. Thirtyfive microliters of diluted samples (1:2 dilution) were incubated overnight on microarray chips (NanoView) coated with capture antibodies for Alix-PE (Green, Biorbyt, St Louis, MO), Syntenin-CF555 (Green, NanoView), CD63-AF647 (Red, NanoView), CD9-AF488 (Blue, NanoView), or negative control IgG1 (NanoView). Once affinity captured, EVs were fixed and permeabilized with the ExoView Cargo Kit to enable access of antibodies to the EVs cargo. Chips were prescanned for background signal and then the conditioned media in incubation solution was dropped onto the chip surface and incubated overnight. The chips were washed, followed by antibody incubation in IF blocking solution. The chips were washed again followed by a rinse in filtered DI water and then dried. The number of positive particles detected for each fluorescence channel and quantified using the ExoView R100 reader using nScan 2.8.4 acquisition software (NanoView). The data were then analysed using NanoViewer 2.8.11 with sizing thresholds set to 50 to $200 \mathrm{~nm}$ diameter.

\section{RNA isolation}

Total RNA, including miRNAs, was isolated using a miRNeasy kit (Qiagen, Hilden, Germany) according to the manufacturer's instructions. The quality of the purified RNA was confirmed by a Nanodrop 2000 
Spectrophotometer and an Agilent 2100 Bioanalyzer (Agilent Technologies, Santa Clara, CA).

\section{NanoString nCounter analysis}

The miRNA profiling analysis was performed by the NanoString nCounter (NanoString Technologies, Inc., Seattle, WA) using 800 human v3 miRNA expression assays according to the manufacturer's protocol. In brief, the NanoString nCounter platform involved mixing total RNA with pairs of capture and reporter probes tailored to each miRNA; this was followed by hybridizing, washing away excess probes, immobilizing probe-bound miRNAs on a surface, and scanning the colour-coded bar tags of the reporter probes. A total of 80-100 ng of total RNA per sample from snap-frozen tissue was used as input material, and $3 \mu \mathrm{l}$ of total volume was used for each sample. All hybridization reactions were incubated at $65{ }^{\circ} \mathrm{C}$ for $18 \mathrm{~h}$, and a max-density scan (555 fields of view) was selected [22].

To identify the molecular subgroups for medulloblastoma, nCounter Elements TagSets was applied as previously described [23]. The algorithm for class prediction analysis was provided by Dr. M. Taylor (Toronto, Canada) and was used for analysis.

\section{mRNA profiling with RNA sequencing analysis}

Total RNA from the BTSCs and BTCs was used for the preparation of paired-end libraries with the TruSeq Stranded mRNA LT Sample Prep Kit. In detail, purified RNAs were randomly fragmented, and the fragmented RNAs were transformed into cDNAs by reverse transcription. The cDNA fragments' both ends were ligated with adaptors. After PCR amplification for a quantity suitable for sequencing, cDNA fragments with insert sizes of 200-400 bp were selected and sequenced in a paired-end manner on a NovaSeq 6000 System.

After trimming the adaptor and removing the lowquality sequences from the raw data, the trimmed RNAseq data were mapped to the human genome (hg19) by using Bowtie 2 and HISAT2 software. Using StringTie software, we performed transcript assembly and calculated Fragments Per Kilobase of transcript per Million mapped reads (FPKM) values.

\section{Analysis of differentially expressed mRNAs and miRNAs}

Using the limma package written in $\mathrm{R}$ programming language (version 3.5.1), we identified differentially expressed genes in the normalized mRNA and miRNA datasets. The mRNAs and miRNAs with a $\log 2-$ fold change $\geq 2$ or $\leq-2$ and a False Discovery Rate $($ FDR $)<0.05$ were considered differentially expressed.

\section{Bioinformatic analysis used to predict target genes} of miRNAs

Using 12 representative miRNA target databases, PhenomiR, DIANA-microT, ElMMo, MicroCosm/miRBase, miRanda, miRDB, PicTar, PITA, TargetScan, miRecords, and miRTarBase, we identified candidate target genes for the differentially expressed miRNAs. To avoid biased prejudice or insufficiency in analysing target genes, we have taken advantage of the comprehensive resources of the above 12 miRNA target databases rather than relying upon one or two individual databases. In choosing the candidate target genes, we maintained the following criteria: either target gene predicted simultaneously by at least 3 of the abovementioned representative miRNA target databases or target gene verified by a research validation in at least one previous investigation.

\section{Integration analysis of miRNAs and mRNAs}

By comparing the abovementioned candidate miRNA target genes and the mRNAs (with log2-fold change, $\geq 2$ or $\leq-2, \mathrm{P}$ value $<0.05$ and False Discovery Rate $[F D R]<0.05)$ in the RNAseq dataset for our samples, we finally selected target genes. To guarantee that we pursued high-quality target genes, we have given a priority (for validation test) to the target genes showing highly statistically significant negative correlation (Pearson correlation coefficient $<-0.7$ ) in expression between miRNAs and mRNAs in our samples. Using the Database for Annotation, Visualization and Integrated Discovery (DAVID) and Kyoto Encyclopedia of Genes and Genomes (KEGG) databases, we performed gene ontology (GO) and pathway analyses for the target genes.

\section{Reverse transcription and quantitative real-time polymerase chain reaction (qRT-PCR)}

Total RNA was extracted, and cDNA was generated using an RT kit (Qiagen) according to the manufacturer's instructions. qRT-PCR was performed to measure selected gene expression by using the ABI real-time PCR detection system and the SYBR Green master mix kit with specific primers. RUN6B was used to normalize cellular miRNA expression. Since there are no known control or housekeeping miRNAs in EVs, we adopted the strategy of using C. elegans miRNAs directly spiked into the Qiazol prior to RNA extraction to be normalizing controls, as previously described [24]. The relative expression of each gene was calculated using the comparative threshold cycle method, as previously described.

\section{In vitro transfection of miRNA inhibitors}

For miRNA inhibitor transfection, BTSCs were grown to $50 \%$ confluency, and then $20 \mathrm{nM}$ of anti-miR-135b and 
anti-miR-135a (Qiagen) were transfected into BTSCs to inhibit their respective miRNAs using the RNAiMAX reagent (ThermoFisher, Waltham, MA) according to the manufacturer's protocol. miScript inhibitor negative control (anti-miR-NC) under the same concentrations and conditions was used as a control. The transfection efficiency was evaluated by qRT-PCR as described above, and all functional studies were performed at $48 \mathrm{~h}$.

\section{Cell viability assay}

The cell viability was assessed after miRNA inhibitor transfection using an EZ-cytox kit (Daeil Lab Service) according to the manufacturer's protocol (All experiments were conducted in triplicate. Cell viability was calculated as the ratio of the absorbance from the miRNA inhibitor-treated cells to the absorbance of the control group cells.

\section{Cell apoptosis assay}

Apoptosis was analyzed by the annexin V-FITC/propidium iodide (PI) apoptosis kit (BD Biosciences, San Jose, $\mathrm{CA}$ ) according to the manufacturer's instructions and as previously described [18]. In brief, the cells were transfected with anti-miRNA, anti-miR-135b or anti-miR$135 \mathrm{a}$ for $48 \mathrm{~h}$ and then the stained cells were detected by FACSCanto (BD), and flow cytometric plots were generated by FlowJo software.

\section{Cell senescence assay}

Senescence-associated $\beta$-galactosidase (SA- $\beta$-gal) activity was detected using the cellular senescence assay kit (Chemicon, Temecula, CA), according to the manufacturer's instructions and as previously described [25]. In brief, the cells were transfected with anti-miRNA, antimiR-135b or anti-miR-135a for $48 \mathrm{~h}$ and then seeded in seeded in 6-well plates, and incubated for $16 \mathrm{~h}$ at $37{ }^{\circ} \mathrm{C}$. Representative microscopic fields were photographed.

\section{Limiting dilution assay}

To assess the self-renewal ability, limiting dilution assays were performed as previously described [17]. After miRNA inhibitor transfection, the cells $\left(1 \times 10^{3}\right)$ were cultured onto 48-well ultra-low cluster plates (Costar). Data were analysed using the extreme limiting dilution software web interface (https://bioinf.wehi.edu.au/softw are/elda/).

Survival analysis and AMOTL2 gene expression association in two paediatric medulloblastoma cohorts

Log-rank tests were performed to examine the association between AMOTL2 expression level and overall survival outcome in two independent paediatric medulloblastoma cohorts from previous studies, including 30 Korean patients (Seoul National University Children's Hospital [SNUCH] cohort) [26] and 530 patients $($ age $<18)$ from Medulloblastoma Advanced Genomic International Consortium (MAGIC) [27, 28]. In the log-rank tests, the patients were divided into high, medium and low AMOTL2 expression groups at one of three cut-off points, $0.25,0.5$, or 0.75 quantile, which were based on the samples in each cohort. Subsequently, survival distributions were compared between the two groups, and the cut-off point with the most significant P-value was selected for each final log-rank test. All analyses were repeated for each medulloblastoma subgroup. Subgrouping of the 30 Korean medulloblastoma cohorts was performed using unsupervised hierarchical clustering and partitioning around medoid (PAM) methods and was based on the expression profiles of 22 marker genes from a CodeSet [29].

\section{Statistical analysis}

All statistical analyses were performed with SPSS 19.0 software (SPSS, Chicago, IL) and GraphPad Prism 5.0 (GraphPad Software, Inc., San Diego, CA). Data are presented as the mean $\pm \mathrm{SD}$. One-way analysis of variance followed by a Tukey post hoc test was used to determine the differences occurring between more than two groups, and a t-test was used to determine the difference between two groups. All experiments were repeated at least three independent times. P-values $<0.05$ were considered statistically significant.

\section{Results}

Characterization of primary cultured cells and EVs from medulloblastoma patients

Prior to all experiments, we performed a characterization analysis of isolated cells and EVs derived from cells, including exosomes. The BTCs and BTSCs cultured under different culture conditions are attached adherent cells and floating spheroid-forming cells, respectively, at passage number 5 (Fig. 1a). The floating BTSCs are considered to be enriched with stem/progenitor cancer cells [17]. We identified the expression of stem cell markers such as nestin and musashi between BTCs and BTSCs. Nestin was expressed in a very small number of cells of BTCs, and no musashi expression was found. On the other hand, BTSCs had very strong expression of both nestin and musashi (Fig. 1b). Immunoblotting showed calnexin expression but no flotillin-1 expression in both BTCs and BTSCs (Fig. 1c).

The characterization of EVs derived from BTCs and BTSCs was confirmed. TEM analysis exhibited round, double-lipid membrane vesicles (Fig. 1d). NTA showed a particle size distribution ranging from 10 to $200 \mathrm{~nm}$ in diameter, with most particles detected in 


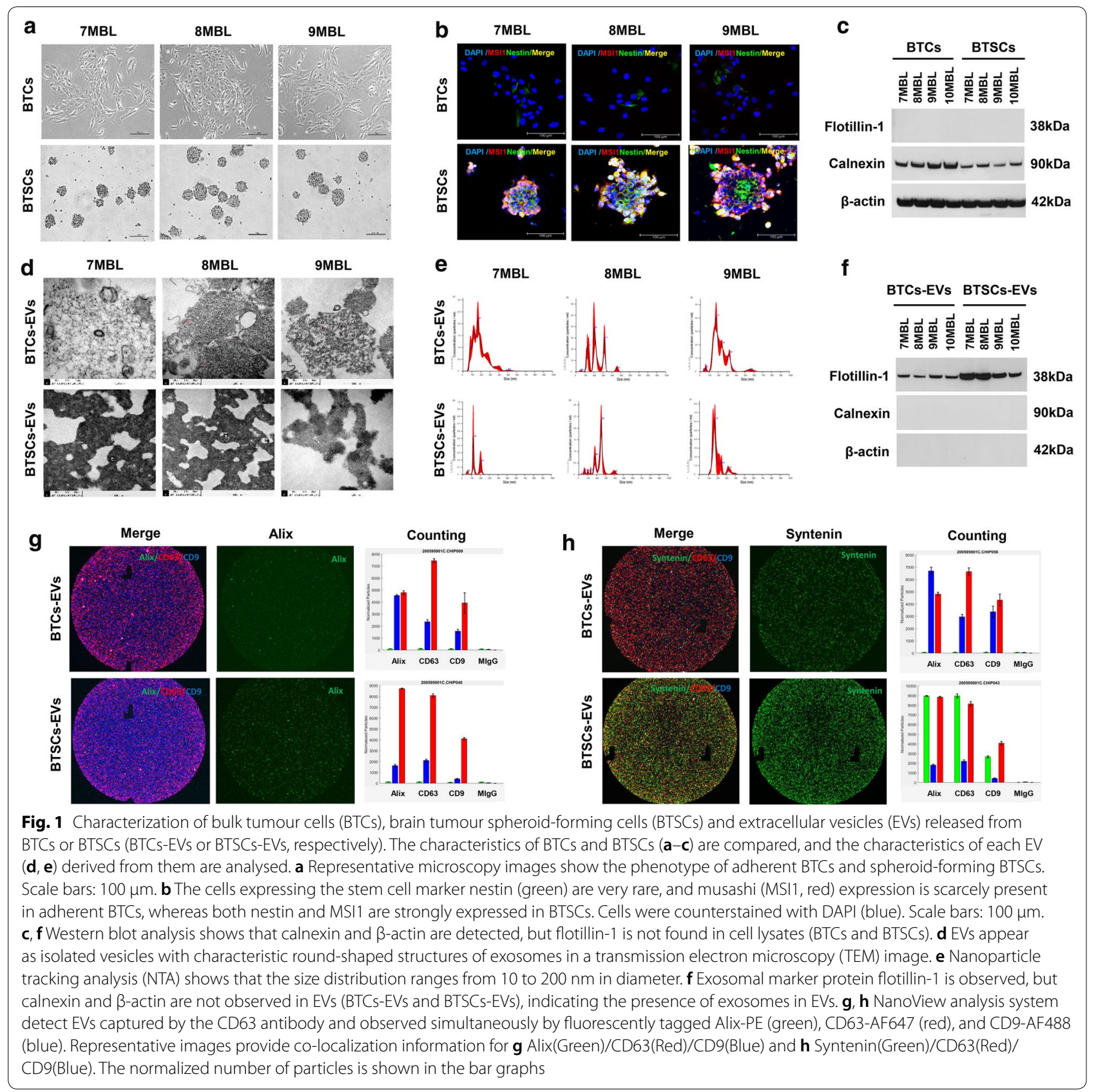

the size range corresponding to exosome dimensions $(85.8 \pm 6.8 \mathrm{~nm}$; Fig. 1e). Immunoblotting revealed the vesicular enrichment of flotillin-1 and the absence of calnexin and $\beta$-actin (Fig. 1f). In addition, ExoView assay provided the presence of internal proteins (Alix and Syntenin) at the EVs level and co-localization with tetraspanins (CD63 and CD9). Immunocaptured vesicles of Alix/CD63/CD9 (Fig. 1g) and Syntenin/CD63/ CD9 (Fig. 1h) were observed in both EVs secreted from BTCs and BTICs.

\section{Comparative analysis of cellular miRNAs and EV-miRNAs between BTCs and BTSCs}

First, we performed gene profiling on 22 subgroup-specific signature genes to identify medulloblastoma subgroups. We confirmed that there were $1 \mathrm{WNT}, 2 \mathrm{SHH}$, 3 Group 3 and 4 Group 4 medulloblastoma through class prediction analysis (Table 1). Next, we performed miRNA profiling of cells and EVs utilizing NanoString nCounter miRNA arrays in individuals with BTSCs compared to those with BTCs. Differentially expressed 
miRNA (DEmiR) analysis showed that 86 miRNAs were differentially expressed with statistical significance in BTSCs compared to BTCs (Fig. 2a, b). In the case of the EVs, 48 miRNAs were differentially expressed in BTSCderived EVs (BTSCs-EVs) compared to in BTC-derived EVs (BTCs-EVs). In both BTSCs-EVs and BTSCs, the expression of 25 miRNAs was found to be highly significantly different, with an interesting observation that 24 of these miRNAs were upregulated compared to in BTCs (Fig. 2c and Table 2). Of these, miR-1246 was upregulated in the BTSCs but downregulated in the BTSCs-EVs. For further functional analysis, we focused on miR-135b and miR-135a, which showed the highest statistical significance and fold change in both BTSCs and BTSCs-EVs. The differential expression of the two miRNAs in BTCs and BTSCs was verified by RT-qPCR (Fig. 2 d, P $<0.001$ ).

\section{Functional regulation of $B T S C s$ by miR-135b or miRNA-135a inhibition}

We next investigated whether secreted miR-135b and miR-135a function at the microenvironment level by possibly impacting the stemness of BTSCs. To determine the effect of miR-135b and miR-135a on the function, mechanism and signal pathway of BTSCs, we conducted in vitro inhibition experiments on Group 4 BTSCs. After anti-miR (miRNA inhibitor) treatment, miR-135b and miR-135a expression levels significantly decreased compared to anti-miR-NC expression levels (Fig. $3 a, \mathrm{P}<0.01$, $\mathrm{P}<0.001)$. Cell viability analysis showed that the cell survival rate was significantly reduced by the inhibition of miR-135b or miR-135a at $48 \mathrm{~h}$ in all BTSCs (Fig. 3b, $\mathrm{P}<0.001$ ). Subsequently, apoptosis and senescence were examined. The early apoptotic rate increased 2.4-5.4 times with anti-miR-135b treatment and 2.5-4.7 times with anti-miR-135a treatment compared with that of the anti-miRNA-NC treatment (Additional file 1: Fig. S1A). However, no differences were observed in senescence (Additional file 1: Fig. S1B). Upon treatment, the sphereforming ability decreased (Fig. 3c), and the expression of nestin and musashi decreased in all of the examined BTSCs (Fig. 3d). Overall, these results suggest that the inhibition of miR-135b or miR-135a can suppress the self-renewal capacity and expression of stem cell-related markers of BTSCs.

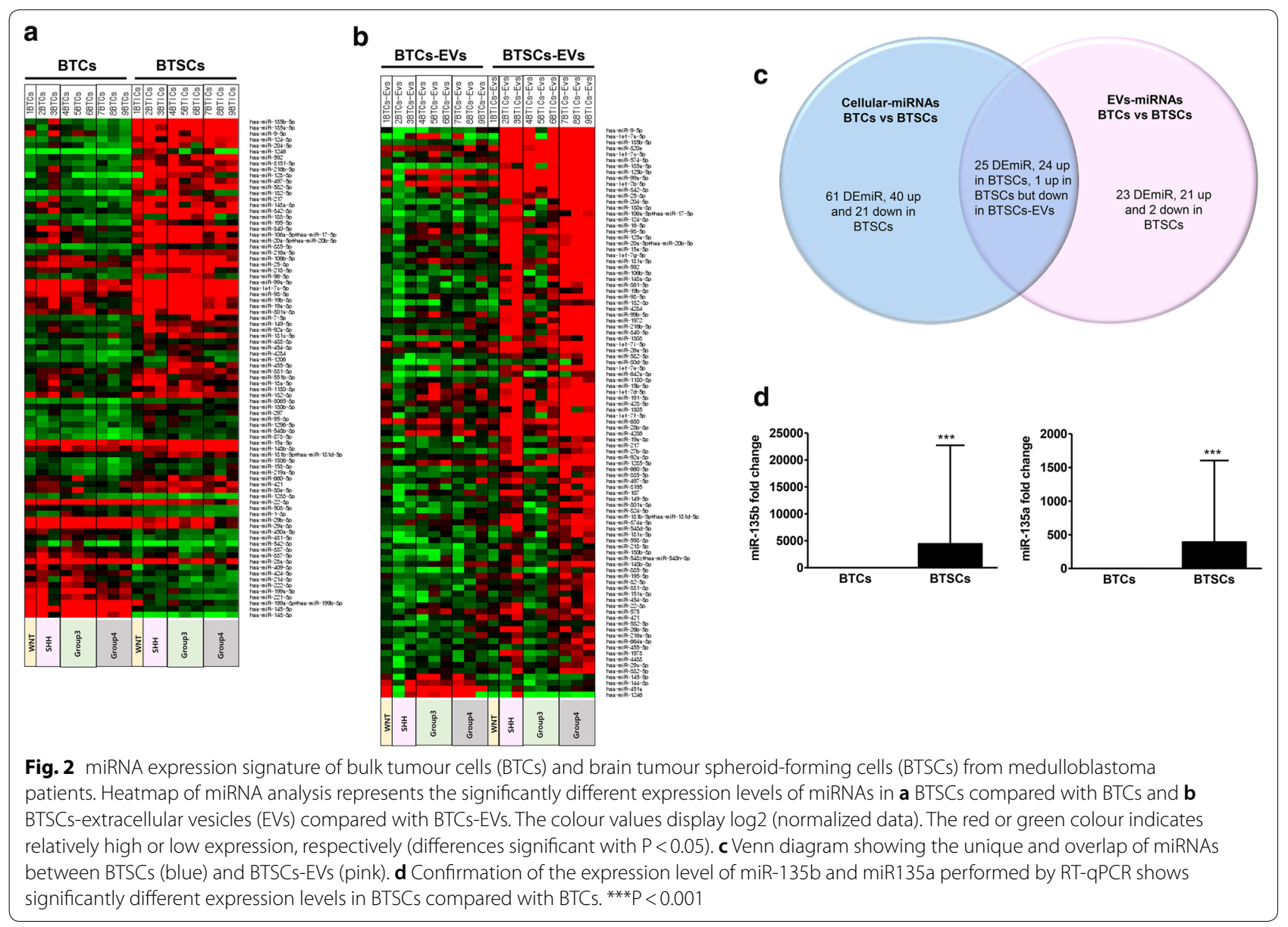


Table 2 Unique and overlapping miRNAs in brain tumor spheroid-forming cells (BTSCs) compared to bulk tumor cells (BTCs)

\begin{tabular}{|c|c|c|c|c|c|}
\hline \multicolumn{2}{|c|}{ Extracellular vesicle (EVs) only } & \multicolumn{2}{|l|}{ Both } & \multicolumn{2}{|l|}{ Cell only } \\
\hline Up-regulated & Down-regulated & Up-regulated & Down-regulated & Up-regulated & Down-regulated \\
\hline hsa-let-7a-5p & hsa-miR-144-3p & hsa-miR-135b-5p & $\begin{array}{l}\text { hsa-miR-1246 (up in } \\
\text { cells, down in EVs) }\end{array}$ & hsa-miR-3151-5p & hsa-miR-1233-3p \\
\hline hsa-miR-320e & hsa-miR-451a & hsa-miR-135a-5p & & hsa-miR-128-3p & hsa-miR-22-3p \\
\hline hsa-miR-574-5p & & hsa-miR-9-5p & & hsa-miR-497-5p & hsa-miR-503-5p \\
\hline hsa-miR-125b-5p & & hsa-miR-124-3p & & hsa-miR-182-5p & hsa-miR-1-3p \\
\hline hsa-let-7b-5p & & hsa-miR-204-5p & & hsa-miR-217 & hsa-miR-29b-3p \\
\hline hsa-miR-130a-3p & & hsa-miR-592 & & hsa-miR-183-5p & hsa-miR-29a-3p \\
\hline hsa-miR-125a-5p & & hsa-miR-216b-5p & & hsa-miR-195-5p & hsa-miR-450a-5p \\
\hline hsa-let-7g-5p & & hsa-miR-582-5p & & hsa-miR-885-5p & hsa-miR-431-5p \\
\hline hsa-miR-181a-5p & & hsa-miR-148a-3p & & hsa-miR-216a-5p & hsa-miR-542-3p \\
\hline hsa-miR-361-5p & & hsa-miR-342-3p & & hsa-miR-218-5p & hsa-miR-337-3p \\
\hline hsa-miR-98-5p & & hsa-miR-340-5p & & hsa-miR-96-5p & hsa-miR-337-5p \\
\hline hsa-miR-99b-5p & & hsa-miR-106a-5p+hsa-miR-17-5p & & hsa-miR-19a-3p & hsa-miR-23a-3p \\
\hline hsa-miR-1972 & & hsa-miR-20a-5p+hsa-miR-20b-5p & & hsa-miR-301a-3p & hsa-miR-409-3p \\
\hline hsa-miR-1303 & & hsa-miR-106b-5p & & hsa-miR-7-5p & hsa-miR-424-5p \\
\hline hsa-let-7i-5p & & hsa-miR-25-3p & & hsa-miR-149-5p & hsa-miR-214-3p \\
\hline hsa-miR-26a-5p & & hsa-miR-99a-5p & & hsa-miR-92a-3p & hsa-miR-222-3p \\
\hline hsa-miR-30d-5p & & hsa-let-7c-5p & & hsa-miR-181c-5p & hsa-miR-199a-5p \\
\hline hsa-let-7e-5p & & hsa-miR-93-5p & & hsa-miR-488-3p & hsa-miR-221-3p \\
\hline hsa-miR-642a-3p & & hsa-miR-19b-3p & & hsa-miR-454-3p & $\begin{array}{l}\text { hsa-miR-199a- } \\
\text { 3p+hsa-miR- } \\
\text { 199b-3p }\end{array}$ \\
\hline hsa-miR-15b-5p & & hsa-miR-4284 & & hsa-miR-1206 & hsa-miR-145-5p \\
\hline hsa-let-7d-5p & & hsa-miR-1180-3p & & hsa-miR-455-5p & hsa-miR-143-3p \\
\hline & & hsa-miR-132-3p & & hsa-miR-331-3p & \\
\hline & & hsa-miR-16-5p & & hsa-miR-551b-3p & \\
\hline & & hsa-miR-15a-5p & & hsa-miR-18a-5p & \\
\hline & & & & hsa-miR-455-3p & \\
\hline & & & & hsa-miR-3065-3p & \\
\hline & & & & hsa-miR-130b-3p & \\
\hline & & & & hsa-miR-297 & \\
\hline & & & & hsa-miR-95-3p & \\
\hline & & & & hsa-miR-1296-5p & \\
\hline & & & & hsa-miR-548b-3p & \\
\hline & & & & hsa-miR-873-5p & \\
\hline & & & & hsa-miR-148b-3p & \\
\hline & & & & $\begin{array}{l}\text { hsa-miR-181b- } \\
\text { 5p+hsa-miR- } \\
\text { 181d-5p }\end{array}$ & \\
\hline & & & & hsa-miR-1306-5p & \\
\hline & & & & hsa-miR-153-3p & \\
\hline & & & & hsa-miR-219a-5p & \\
\hline & & & & hsa-miR-660-5p & \\
\hline & & & & hsa-miR-421 & \\
\hline & & & & hsa-miR-30e-5p & \\
\hline
\end{tabular}




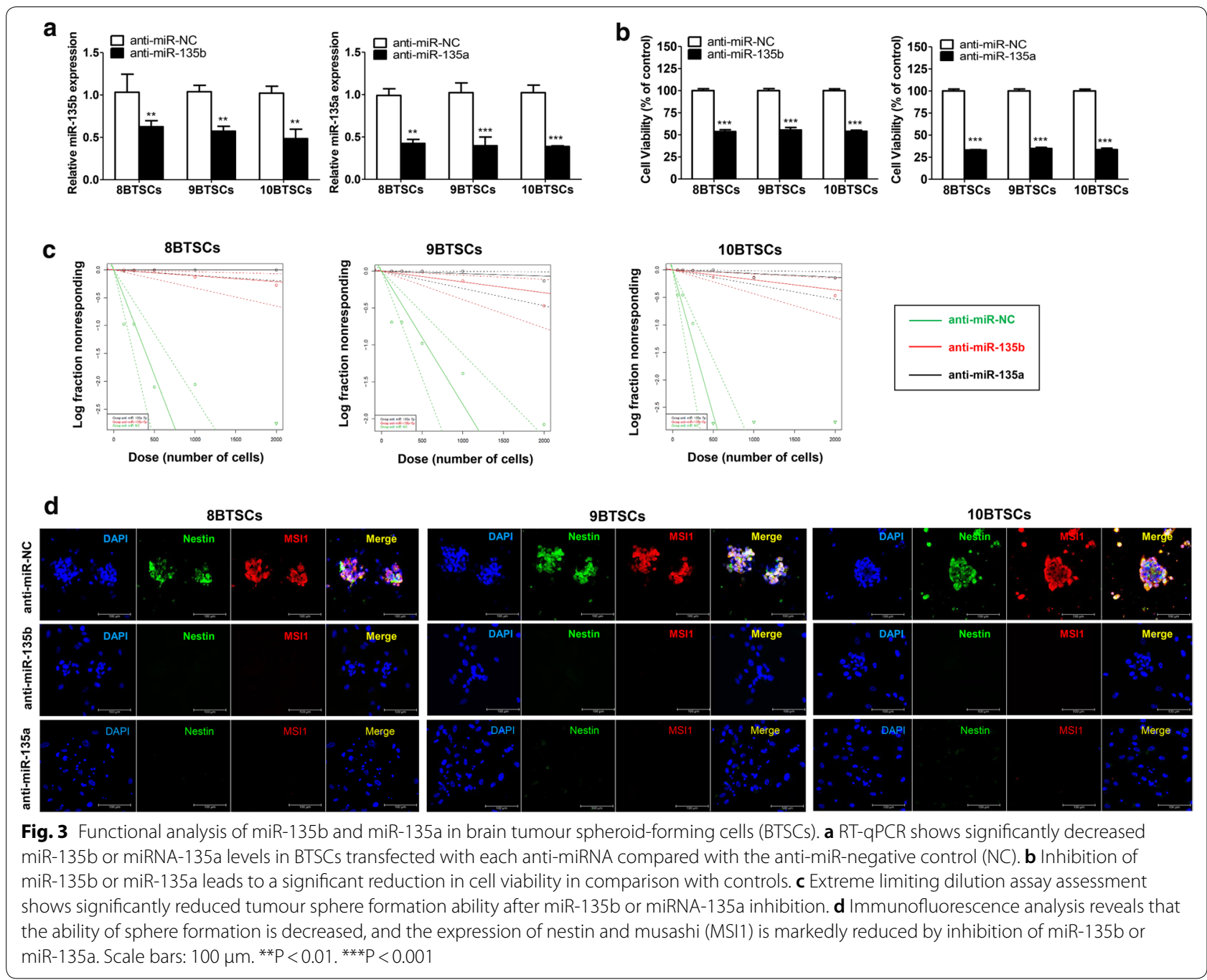

\section{Integrative analysis of miRNA and mRNA expression with predicted miRNA targets}

We analysed the predicted target mRNAs of the upregulated miRNAs with downregulated mRNA expression from the microarray and vice versa [30-33]. Based on the above method, a total of 325 significant miRNAmRNA pairs were predicted, consisting of 109 differentially expressed miRNAs and 1640 mRNAs. Among the 750 significantly decreased mRNAs, 199 genes were predicted to be targets of 23 upregulated miRNAs (Fig. 4a). The target mRNA of miR-135b and/or miR-135a was selected, verified and analysed $(\mathrm{N}=10$, Fig. 4b, $\mathrm{P}<0.001)$. We found that miR-135b targets only one gene, AMOTL2, and miR-135a targets AMOTL2, signal transducer and activator of transcription 6
(STAT6) and glutathione peroxidase 8 (GPX8). Notably, AMOTL2 was targeted by both miR-135b and miR-135a. AMOTL2 expression levels were negatively correlated with those of miR-135b $(R=-0.92006$; $\mathrm{P}<0.0001)$ and miR-135a $(\mathrm{R}=-0.82885 ; \mathrm{P}<0.0001)$ (Fig. 5a, b, Additional file 2: Fig. S2A and S2B). STAT6 $(\mathrm{R}=-0.85386 ; \mathrm{P}<0.0001)$ and GPX8 $(\mathrm{R}=-0.81841$; $\mathrm{P}<0.0001$ ) were also associated with miR-135a (Fig. 5a, $\mathrm{b}$ and Additional file 2: Fig. S2C). Although the number of samples was small, there were no significant differences according to subgroups, and their association's patterns were found to be similar in all groups. These results implied that high expression levels of miR-135b and miR-135a target and downregulate the expression of AMOTL2. To further determine whether AMOTL2 


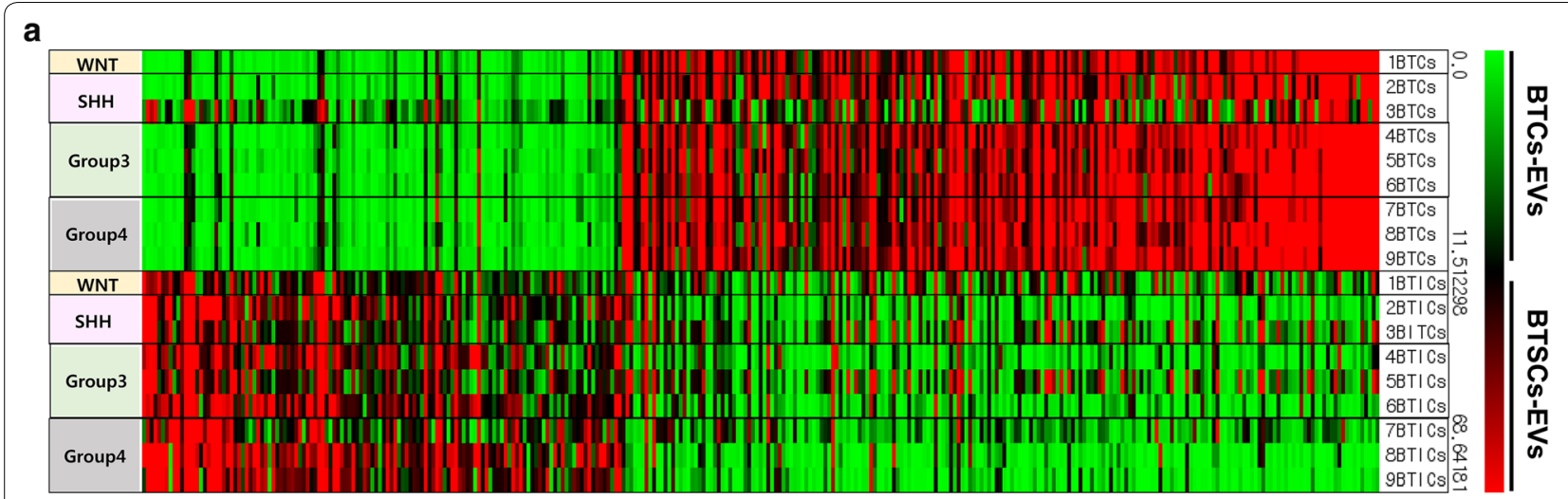

b
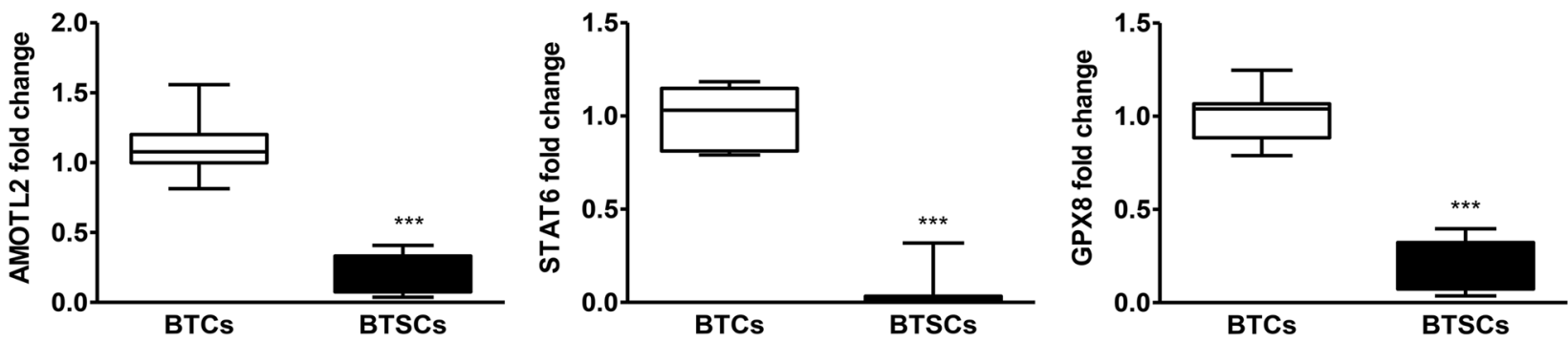

Fig. 4 Integrated analysis and validation of mRNA-targeted miRNAs in brain tumour spheroid-forming cells (BTSCs). a Differential gene expression between bulk tumour cells (BTCs) and BTSCs was determined by RNA-seq. According to the integrated analysis, there are 199 genes that are predicted to be targets of the 23 upregulated miRNAs. The number of previously identified targets for miR-135b and miR-135a targets is 1 and 3 , respectively. $\mathbf{b}$ The predicted target genes for miR-135b or miR-135a were identified as AMOTL2, STAT6, GPX8, and their expression was verified by RT-qPCR. Compared with BTCs, the expression of AMOTL2, STAT6, and GPX8 was observed to be significantly reduced in BTSCs. ${ }^{* *}$ P $<0.001$

is regulated by miR-135b or miR-135a, the expression level of AMOTL2 after inhibition of miR-135b and miR-135a was investigated by RT-qPCR. The expression of AMOTL2 was 15.7- and 5.3-fold higher following inhibition of miR-135b and miR-135a, respectively, when compared with the control (Fig. 5c). These results show that miR-135a and miR-135a function in BTSCs, at least partially through down-regulating the expression of AMOTL2.

To explore the biological pathway associated with miR135b-targeted AMOTL2, we used KEGG pathway analysis. As a result, we found the Hippo and tight junction pathways involved in AMOTL2. Based on our RNA-seq results, we identified upregulated (red) and downregulated (green) genes (Additional file 3: Fig. S3).

\section{Association of AMOTL2 expression with overall survival outcome}

We evaluated the association between the expression level of AMOTL2 and clinical outcome in two independent cohorts (SNUCH and MAGIC) [26-28]. Survival analysis revealed that high expression of AMOTL2 was significantly associated with a favourable prognosis in paediatric medulloblastoma patients in Group 3 and Group 4, which was consistently observed in the two independent cohorts (Fig. 6). Unlike Group 3 and Group 4, high expression of AMOTL2 was associated with poor prognosis in WNT, and there was no significant correlation in $\mathrm{SHH}$ group.

\section{Discussion}

In this study, we profiled both cellular miRNAs and EVmiRNAs from BTCs and BTSCs and found that miR$135 \mathrm{~b}$ and miR-135a were highly expressed in the BTSCs and BTSCs-EVs. Importantly, inhibition of miR-135b and miR-135a reduces the stemness of medulloblastoma BTSCs. Furthermore, an integrated analysis suggested that miR-135b and miR-135a regulate AMOTL2. Our results might be the first evidence to demonstrate that the secretion of these miRNAs through EVs could potentially be a consequence of BTSCs attempting to maintain their stemness or signalling to other cells.

Previous evidence has indicated that miR-135b is highly expressed in the CSCs of paediatric solid tumours [34, 35]. The direct inhibition of miR-135b may itself serve 

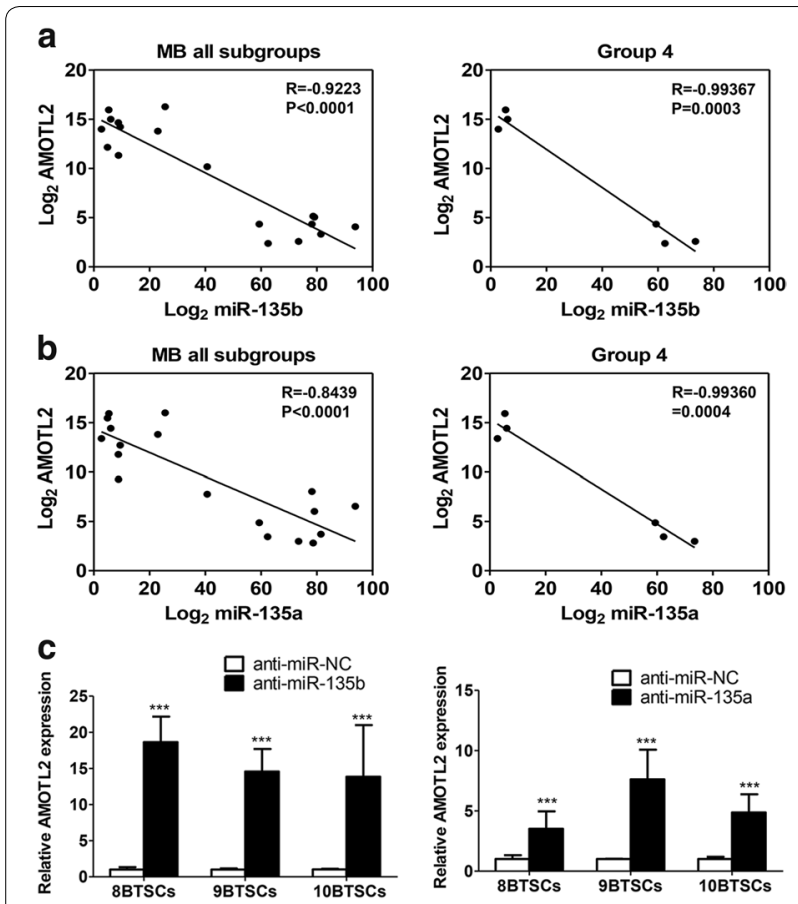

Fig. 5 The correlation coefficients between target genes AMOTL2 and miR-135b and miR-135a in medulloblastoma all subgroups and Group 4. a The R value of AMOTL2, the target of both miR-135b and miR-135a, is $-0.92006(P<0.0001)$ for miR-135b and -0.82885 $(P<0.0001)$ for miR-135a. The $R$ values are also shown for each subgroup of medulloblastoma. $\mathbf{b}$ RT-qPCR reveal increased expression of AMOTL2 in BTSCs after miR-135b or miR-135a inhibition. ${ }^{* * *} \mathrm{P}<0.001$

as a therapeutic intervention against a network of events driving oncogenesis and may even target self-renewing CSCs [35]. In the case of miR-135a, there has been controversy over whether onco-miRs or tumour suppressormiRs are present in several other tumours. miR-135a is known as onco-miR in breast cancer [36] and colorectal carcinomas [37], but it is known as tumour suppressor in renal cell carcinoma [38] and gastric cancer [39]. The opposite function of miR-135a was identified in different genetic contexts in a study of glioblastoma [40]. This controversy may also be due to the difference between the analysed sample and the comparison group.

Based on the results of our analysis, we conducted functional verification focusing on $\mathrm{miR}-135 \mathrm{~b}$ and $\mathrm{miR}-$ 135 a by focusing on the highest log fold-change and the p-value. Our stemness assay confirmed that in vitro blockade of miR-135b or miR-135a demonstrated a markedly diminished ability of the medulloblastoma cells to form spheres, thereby alluding to the role of miR-135b and miR-135a in self-renewal and proliferation.
AMOTL2 belongs to the angiomotin (AMOT) family of membrane-associated scaffold proteins. AMOTL2 is commonly accepted as a tumour suppressor [41, 42]. AMOTL2 has been shown to regulate cell proliferation and migration and is involved in the control of the Hippo pathway $[43,44]$. In particular, the Hippo pathway of CSCs plays a significant role in tumorigenesis, chemoresistance, metastatic potential, and self-renewal by promoting CSC characteristics [45, 46]. However, the CSC-specific regulatory mechanisms of the Hippo pathway and AMOTL2 [45] remain unclear, and signalling through CSC-secreted EVs has not been elucidated. Notably, we demonstrated that miR-135b and miR-135a targeted AMOTL2 and the expression of AMOTL2 can be increased through miR-135b and miR-135a inhibition. This result might be a clue that miR-135b and miR-135a of BTSCs-EVs may be able to regulate the Hippo pathway via AMOTL2, a major molecule of the Hippo pathway.

In addition, we observed that high expression of AMOTL2 is associated with prolonged survival in two paediatric medulloblastoma cohorts, particularly in the patients of Group 3 and Group 4. The expression of AMOTL2 may be implicated in the prognosis of certain subgroups. This result also suggests a possible role of AMOTL2 as a tumour suppressor in medulloblastoma Groups 3 and 4.

The limitation of our study is the small number of specimens, which not only is based on a relatively limited incidence of paediatric medulloblastoma but also reflects the difficulty in simultaneously isolating spheroid-forming cells and bulk tumour cells for acquiring a sufficient amount of EVs. Future research is necessary to extend the significance of our findings and to more deeply explore the specific contributions of each miRNA identified here. If the regulation of miR-135b and miR-135a affects the stemness properties of BTSCs, in vivo experiments are required to validate the relevance between the regulation of these miRNAs and tumour progression.

\section{Conclusion}

The present study described the miRNA expression pattern in medulloblastoma BTSCs and EVs derived from BTSCs, as well as their potential role in affecting recipient cells. The study provides new insights into the epigenetic regulation of CSCs in medulloblastoma. These findings may act as a basis for further studies into the discovery of better prognostic markers and the development of promising therapeutics, as well as for deciphering the specific role of EVs in CSC maintenance, regulation and progression. 


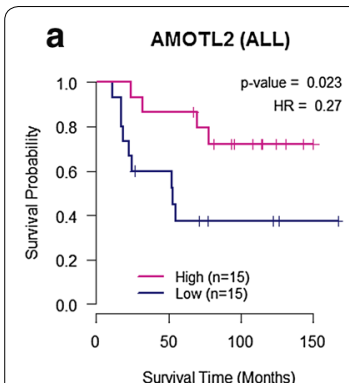

b AMOTL2 (ALL)

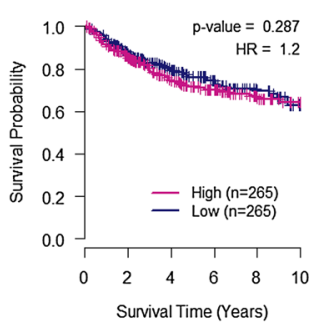

AMOTL2 (SHH)

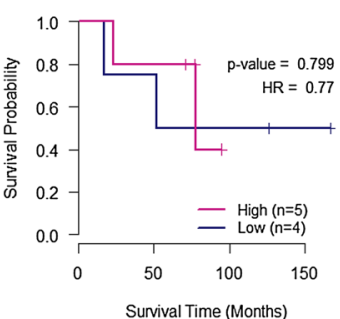

AMOTL2 (Group 3)

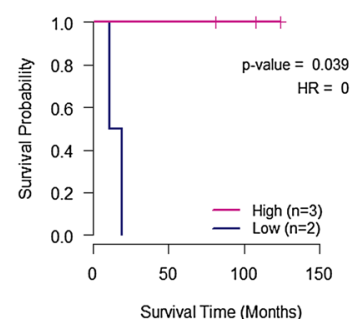

AMOTL2 (Group 4)

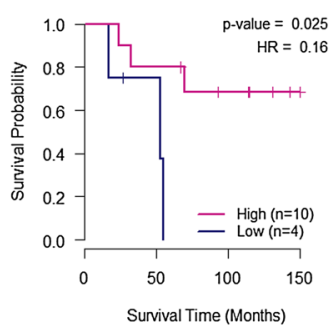

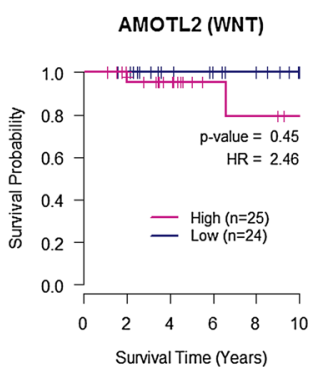
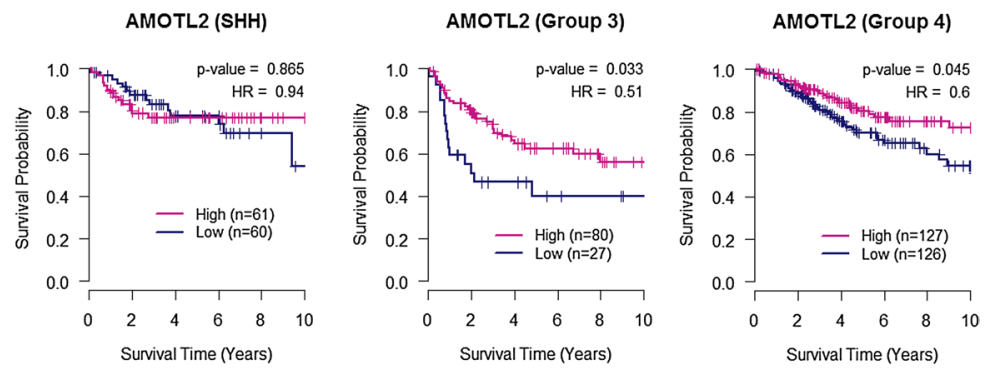

Fig. 6 Association analysis between the expression level of AMOTL2 and the overall survival of paediatric medulloblastoma patients in two independent cohorts. a Kaplan-Meier plots with log-rank tests in two groups of 30 Korean patients with high or low expression of AMOTL2 (Seoul National University Children's Hospital [SNUCH] cohort). b Kaplan-Meier plots with log-rank tests in two groups of patients with high or low expression of AMOTL2 in the Medulloblastoma Advanced Genomics International Consortium (MAGIC) cohort

\section{Supplementary information}

Supplementary information accompanies this paper at https://doi. org/10.1186/s12935-020-01645-6.

Additional file 1: Fig. S1. Apoptosis and senescence analysis by ani-miR135b and anti-miR-135a treatment in brain tumour spheroid-forming cells (BTSCs). (A) Flow cytometric analysis is applied to determine the ratio of apoptosis with Annexin V-FITC/PI staining. The anti-miR-135b and antimiR-135a treatment induce the early apoptosis cells compared with antimiR-NC. (B) Representative images of SA- $\beta$-gal staining after ani-miR-135b and anti-miR-135a treatment display no senescence induced cells.

Additional file 2: Fig. S2. The correlation coefficients between target genes AMOTL2 and (A) miR-135b and (B) miR-135a in medulloblastoma subgroups of WNT, SHH and Group 3. (C) The R values of STAT6 and GPX8, which are targeted only to miR-135a, were $-0.85386(P<0.0001)$ and $-0.81841(P<0.0001)$, respectively.

Additional file 3: Fig. S3. Visualization of AMOTL2 expression pattern in the Hippo signalling pathway and in the tight junction pathway in brain tumour spheroid-forming cells (BTSCs).

\section{Acknowledgements}

We thank all the contributors for this work.

\section{Authors' contributions}

SKK and SAC designed the study. SAC conducted the main experiments. EJK, JWB, JHP, JY, DWH and JYL carried out partial experiments. RNK and AKP carried out data analyses. SC wrote the manuscript draft. EJK and KCW revised the manuscript. All authors read and approved the final manuscript.

\section{Funding}

This work (2017R1A2B2008422) was supported by the Mid-career Researcher Program through an NRF grant funded by the Korean government (Ministry of Science and ICT).

\section{Availability of data and materials}

The datasets generated and analyzed during the current study are available from the corresponding author upon reasonable request.

\section{Ethics approval and consent to participate}

This research for using human samples was reviewed and approved by the Institutional Review Board (IRB) of the Seoul National University Hospital.

\section{Consent for publication}

The publication of this manuscript has been approved by all authors.

\section{Competing interests}

The authors declare that they have no competing interests.

\section{Author details}

1 Division of Pediatric Neurosurgery, Pediatric Clinical Neuroscience Center, Seoul National University Children's Hospital, 101 Daehak-ro, Jongno-gu, Seoul 03080, Republic of Korea. ${ }^{2}$ Department of Neurosurgery, Seoul National University Hospital, Seoul National University College of Medicine, Seoul, Korea. ${ }^{3}$ Regional Emergency Medical Center, Seoul National University Hospital, Seoul, Korea. ${ }^{4}$ Department of Biomedical Engineering, Seoul National University, Seoul, Korea. ${ }^{5}$ Department of Nuclear Medicine, Seoul National University College of Medicine, Seoul, Korea. ${ }^{6}$ College of Pharmacy and Research Institute of Life and Pharmaceutical Sciences, Sunchon National University, Suncheon, Korea. ${ }^{7}$ Department of Anatomy, Neural Development and Anomaly Lab, Seoul National University College of Medicine, Seoul, Korea.

Received: 4 June 2020 Accepted: 9 November 2020

Published online: 20 November 2020 


\section{References}

1. Plaks V, Kong N, Werb Z. The cancer stem cell niche: how essential is the niche in regulating stemness of tumor cells? Cell Stem Cell. 2015;16(3):225-38.

2. Anido J, Saez-Borderias A, Gonzalez-Junca A, Rodon L, Folch G, Carmona MA, Prieto-Sanchez RM, Barba I, Martinez-Saez E, Prudkin $\mathrm{L}$, et al. TGF-beta receptor inhibitors target the CD44(high)/Id1(high) glioma-initiating cell population in human glioblastoma. Cancer Cell. 2010:18(6):655-68.

3. Ishiguro T, Ohata H, Sato A, Yamawaki K, Enomoto T, Okamoto K. Tumorderived spheroids: relevance to cancer stem cells and clinical applications. Cancer Sci. 2017;108(3):283-9.

4. Wang J, Zheng Y, Zhao M. Exosome-based cancer therapy: implication for targeting cancer stem cells. Front Pharmacol. 2016;7:533.

5. Huang GH, Xu QF, Cui YH, Li N, Bian XW, Lv SQ. Medulloblastoma stem cells: promising targets in medulloblastoma therapy. Cancer Sci. 2016;107(5):583-9.

6. Manoranjan B, Venugopal C, McFarlane N, Doble BW, Dunn SE, Scheinemann K, Singh SK. Medulloblastoma stem cells: where development and cancer cross pathways. Pediatr Res. 2012;71 (4 Pt 2):516-22

7. Polkinghorn WR, Tarbell NJ. Medulloblastoma: tumorigenesis, current clinical paradigm, and efforts to improve risk stratification. Nat Clin Pract Oncol. 2007:4(5):295-304

8. Boman KK, Hoven E, Anclair M, Lannering B, Gustafsson G. Health and persistent functional late effects in adult survivors of childhood CNS tumours: a population-based cohort study. Eur J Cancer. 2009;45(14):2552-61.

9. D'Asti E, Chennakrishnaiah S, Lee TH, Rak J. Extracellular vesicles in brain tumor progression. Cell Mol Neurobiol. 2016;36(3):383-407.

10. Ciregia F, Urbani A, Palmisano G. Extracellular vesicles in brain tumors and neurodegenerative diseases. Front Mol Neurosci. 2017;10:276.

11. Becker A, Thakur BK, Weiss JM, Kim HS, Peinado H, Lyden D. Extracellular vesicles in cancer: cell-to-cell mediators of metastasis. Cancer Cell. 2016;30(6):836-48

12. Colombo M, Raposo G, Thery C. Biogenesis, secretion, and intercellular interactions of exosomes and other extracellular vesicles. Annu Rev Cell Dev Biol. 2014:30:255-89.

13. Bronisz A, Godlewski J, Chiocca EA. Extracellular vesicles and MicroRNAs: their role in tumorigenicity and therapy for brain tumors. Cell Mol Neurobiol. 2016;36(3):361-76

14. Jiang X, Hu S, Liu Q, Qian C, Liu Z, Luo D. Exosomal microRNA remodels the tumor microenvironment. PeerJ. 2017;5:e4196.

15. Wu J, Qu Z, Fei ZW, Wu JH, Jiang CP. Role of stem cell-derived exosomes in cancer. Oncol Lett. 2017;13(5):2855-66.

16. Epple LM, Griffiths SG, Dechkovskaia AM, Dusto NL, White J, Ouellette RJ, Anchordoquy TJ, Bemis LT, Graner MW. Medulloblastoma exosome proteomics yield functional roles for extracellular vesicles. PLOS ONE. 2012;7(7):e42064

17. Choi SA, Lee JY, Phi JH, Wang KC, Park CK, Park SH, Kim SK. Identification of brain tumour initiating cells using the stem cell marker aldehyde dehydrogenase. Eur J Cancer. 2014;50(1):137-49.

18. Phi JH, Choi SA, Kim SK, Wang KC, Lee JY, Kim DG. Overcoming chemoresistance of pediatric ependymoma by inhibition of STAT3 signaling. Transl Oncol. 2015:8(5):376-86.

19. Huang H, Zheng X, Cai C, Yao Z, Lu S, Meng X, Miao Y, He Z, Cai C, Zou F. Exosomes derived from breast cancer lung metastasis subpopulations promote tumor self-seeding. Biochem Biophys Res Commun. 2018;503(1):242-8.

20. Hoshino A, Kim HS, Bojmar L, Gyan KE, Cioffi M, Hernandez J, Zambirinis CP, Rodrigues G, Molina H, Heissel S, et al. Extracellular vesicle and particle biomarkers define multiple human cancers. Cell. 2020;182(4):1044-1061. e1018.

21. Bachurski D, Schuldner M, Nguyen PH, Malz A, Reiners KS, Grenzi PC, Babatz F, Schauss AC, Hansen HP, Hallek M, et al. Extracellular vesicle measurements with nanoparticle tracking analysis - an accuracy and repeatability comparison between NanoSight NS300 and ZetaView. J Extracell Vesicles. 2019:8(1):1596016.

22. Bhagirath D, Yang TL, Bucay N, Sekhon K, Majid S, Shahryari V, Dahiya R, Tanaka Y, Saini S. microRNA-1246 is an exosomal biomarker for aggressive prostate cancer. Cancer Res. 2018;78(7):1833-44.
23. Lee C, Lee J, Choi SA, Kim SK, Wang KC, Park SH, Kim SH, Lee JY, Phi JH. M1 macrophage recruitment correlates with worse outcome in $\mathrm{SHH}$ medulloblastomas. BMC Cancer. 2018:18(1):535.

24. Pfeffer SR, Grossmann KF, Cassidy PB, Yang CH, Fan M, Kopelovich L, Leachman SA, Pfeffer LM. Detection of exosomal miRNAs in the plasma of melanoma patients. J Clin Med. 2015;4(12):2012-27.

25. Alessio N, Aprile D, Squillaro T, Di Bernardo G, Finicelli M, Melone MA Peluso G, Galderisi U. The senescence-associated secretory phenotype (SASP) from mesenchymal stromal cells impairs growth of immortalized prostate cells but has no effect on metastatic prostatic cancer cells. Aging. 2019;11(15):5817-28.

26. Park AK, Lee SJ, Phi JH, Wang KC, Kim DG, Cho BK, Haberler C, Fattet S, Dufour C, Puget $\mathrm{S}$, et al. Prognostic classification of pediatric medulloblastoma based on chromosome 17p loss, expression of MYCC and MYCN, and Wnt pathway activation. Neuro Oncol. 2012;14(2):203-14.

27. Park AK, Lee JY, Cheong H, Ramaswamy V, Park SH, Kool M, Phi JH, Choi SA Cavalli F, Taylor MD, et al. Subgroup-specific prognostic signaling and metabolic pathways in pediatric medulloblastoma. BMC Cancer. 2019;19(1):571.

28. Cavalli FMG, Remke M, Rampasek L, Peacock J, Shih DJH, Luu B, Garzia L, Torchia J, Nor C, Morrissy AS, et al. Intertumoral heterogeneity within medulloblastoma subgroups. Cancer Cell. 2017;31(6):737-754.e736.

29. Northcott PA, Shih DJ, Remke M, Cho YJ, Kool M, Hawkins C, Eberhart CG, Dubuc A, Guettouche T, Cardentey Y, et al. Rapid, reliable, and reproducible molecular sub-grouping of clinical medulloblastoma samples. Acta Neuropathol. 2012;123(4):615-26.

30. Huang JC, BabakT, Corson TW, Chua G, Khan S, Gallie BL, Hughes TR, Blencowe BJ, Frey BJ, Morris QD. Using expression profiling data to identify human microRNA targets. Nat Methods. 2007;4(12):1045-9.

31. Dmitriev P, Barat A, Polesskaya A, O'Connell MJ, Robert T, Dessen P, Walsh TA, Lazar $\vee$, Turki A, Carnac G, et al. Simultaneous miRNA and mRNA transcriptome profiling of human myoblasts reveals a novel set of myogenic differentiation-associated miRNAs and their target genes. BMC Genomics. 2013;14:265.

32. Moraes LN, Fernandez GJ, Vechetti-Junior IJ, Freire PP, Souza RWA, Villacis RAR, Rogatto SR, Reis PP, Dal-Pai-Silva M, Carvalho RF. Integration of miRNA and mRNA expression profiles reveals microRNA-regulated networks during muscle wasting in cardiac cachexia. Sci Rep. 2017;7(1):6998.

33. Wang YP, Li KB. Correlation of expression profiles between microRNAs and mRNA targets using NCl-60 data. BMC Genomics. 2009;10:218.

34. Sanchez-Diaz PC, Hsiao TH, Chang JC, Yue D, Tan MC, Chen HI, Tomlinson GE, Huang $Y, C$ Chen $Y$, Hung JY. De-regulated microRNAs in pediatric cancer stem cells target pathways involved in cell proliferation, cell cycle and development. PLoS ONE. 2013;8(4):e61622.

35. Khatri R, Subramanian S. MicroRNA-135b and its circuitry networks as potential therapeutic targets in colon cancer. Front Oncol. 2013;3:268.

36. Chen Y, Zhang J, Wang H, Zhao J, Xu C, Du Y, Luo X, Zheng F, Liu R, Zhang H, et al. miRNA-135a promotes breast cancer cell migration and invasion by targeting HOXA10. BMC Cancer. 2012;12:111.

37. Nagel R, le Sage C, Diosdado B, van der Waal M, Oude Vrielink JA, Bolijn A, Meijer GA, Agami R. Regulation of the adenomatous polyposis coli gene by the miR-135 family in colorectal cancer. Cancer Res. 2008;68(14):5795-802.

38. Hidaka H, Seki N, Yoshino H, Yamasaki T, Yamada Y, Nohata N, Fuse M, Nakagawa M, Enokida $\mathrm{H}$. Tumor suppressive microRNA-1285 regulates novel molecular targets: aberrant expression and functional significance in renal cell carcinoma. Oncotarget. 2012;3(1):44-57.

39. Wu H, Huang M, Cao P, Wang T, Shu Y, Liu P. MiR-135a targets JAK2 and inhibits gastric cancer cell proliferation. Cancer Biol Ther. 2012;13(5):281-8.

40. Cheng Z, Liu F, Zhang H, Li X, Li Y, Li J, Liu F, Cao Y, Cao L, Li F. miR-135a inhibits tumor metastasis and angiogenesis by targeting FAK pathway. Oncotarget. 2017:8(19):31153-68.

41. Mojallal M, Zheng Y, Hultin S, Audebert S, van Harn T, Johnsson P, Lenander C, Fritz N, Mieth C, Corcoran M, et al. AmotL2 disrupts apical-basal cell polarity and promotes tumour invasion. Nat Commun. 2014;5:4557.

42. Ehmer U, Sage J. Control of proliferation and cancer growth by the hippo signaling pathway. Mol Cancer Res. 2016;14(2):127-40.

43. Yi C, Troutman S, Fera D, Stemmer-Rachamimov A, Avila JL, Christian N, Persson NL, Shimono A, Speicher DW, Marmorstein R, et al. A tight junction-associated Merlin-angiomotin complex mediates Merlin's regulation of mitogenic signaling and tumor suppressive functions. Cancer Cell. 2011;19(4):527-40. 
44. Aase K, Ernkvist M, Ebarasi L, Jakobsson L, Majumdar A, Yi C, Birot O, Ming Y, Kvanta A, Edholm D, et al. Angiomotin regulates endothelial cell migration during embryonic angiogenesis. Genes Dev. 2007;21(16):2055-68.

45. Park JH, Shin JE, Park HW. The role of Hippo pathway in cancer stem cell biology. Mol Cells. 2018;41(2):83-92.

46. Hong L, Li Y, Liu Q, Chen Q, Chen L, Zhou D. The hippo signaling pathway in regenerative medicine. Methods Mol Biol. 2019;1893:353-70.

47. Chang CH, Housepian EM, Herbert C Jr. An operative staging system and a megavoltage radiotherapeutic technic for cerebellar medulloblastomas. Radiology. 1969;93(6):1351-9.

\section{Publisher's Note}

Springer Nature remains neutral with regard to jurisdictional claims in published maps and institutional affiliations.
Ready to submit your research? Choose BMC and benefit from:

- fast, convenient online submission

- thorough peer review by experienced researchers in your field

- rapid publication on acceptance

- support for research data, including large and complex data types

- gold Open Access which fosters wider collaboration and increased citations

- maximum visibility for your research: over $100 \mathrm{M}$ website views per year

At BMC, research is always in progress.

Learn more biomedcentral.com/submissions 